\title{
3 Research Square

\section{Renal Resistive Index on Intensive Care Unit Admission Correlates with Tissue Hypoperfusion Indices and Predicts Clinical Outcome}

\section{Georgia Fotopoulou}

Medical School, National and Kapodistrian University of Athens

Ioannis Poularas

Medical School, National and Kapodistrian University of Athens

\section{Stelios Kokkoris}

Medical School, National and Kapodistrian University of Athens

\section{Efstratia Charitidou}

Medical School, National and Kapodistrian University of Athens

\section{loannis Boletis}

Medical School, National and Kapodistrian University of Athens

\section{Elias Brountzos}

Medical School, National and Kapodistrian University of Athens

\section{Athanasios Benetos}

Department of Geriatrics CHRU Nancy

\section{Spyros Zakynthinos}

Medical School, National and Kapodistrian University of Athens

Christina Routsi ( $\nabla$ chroutsi@hotmail.com )

National and Kapodistrian University of Athens School of Health Sciences: Ethniko kai Kapodistriako Panepistemio Athenon https://orcid.org/0000-0002-5871-1361

\section{Research}

Keywords: Renal Doppler ultrasonography, acute kidney injury, renal resistive index, sepsis, shock, intensive care, tissue hypoxia, central venous-to-arterial carbon dioxide tension difference by arterial-tocentral venous oxygen content difference $(\mathrm{P}(\mathrm{cv}-\mathrm{a}) \mathrm{CO} 2 / \mathrm{C}(\mathrm{a}-\mathrm{cv}) 02)$, lactate

Posted Date: August 23rd, 2021

DOI: https://doi.org/10.21203/rs.3.rs-806081/v1

License: (c) (i) This work is licensed under a Creative Commons Attribution 4.0 International License.

Read Full License 
Version of Record: A version of this preprint was published at Shock on December 3rd, 2021. See the published version at https://doi.org/10.1097/SHK.0000000000001896. 


\section{Abstract}

Background: Recent advancements in the context of shock pathophysiology, support ultrasound assessment of organ perfusion. Renal resistive index (RRI) has been used to evaluate renal blood flow. Our aim was to investigate the relation between RRI, and global tissue hypoperfusion indices, in mechanically ventilated critically ill patients and their association with clinical outcome.

Methods: In this prospective observational study, RRI was measured within 24 hours of intensive care unit (ICU) admission. Clinical and laboratory data, routine hemodynamic variables and gas exchange at the time of RRI assessment were recorded. The ratio of central venous-to-arterial carbon dioxide partial pressure difference by arterial-to-central venous oxygen content difference $\left(\mathrm{P}_{(\mathrm{cv}-\mathrm{a})} \mathrm{CO}_{2} / \mathrm{C}_{(\mathrm{a}-\mathrm{cv})} \mathrm{O}_{2}\right)$ and lactate were used as global tissue hypoperfusion indices.

Results A total of 126 mechanically ventilated patients were included [median age 61 (IQR 28) years, 74\% males]. Seventy-seven patients had RRI values $>0.7 . \mathrm{P}_{(\mathrm{cv}-\mathrm{a})} \mathrm{CO}_{2} / \mathrm{C}_{(\mathrm{a}-\mathrm{cv})} \mathrm{O}_{2}$ ratio and arterial lactate, were significantly higher in patients with $\mathrm{RRI}>0.7$ compared to those with $\mathrm{RRI} \leq 0.7$ [2.4 (2.2) versus 1.2 (0.6) and 2.88 (3.39) versus $0.62(0.57) \mathrm{mmol} / \mathrm{I}$ respectively, both $\mathrm{p}<0.001)$ ]. RRI was significantly correlated with $\mathrm{P}_{(\mathrm{cv}-\mathrm{a})} \mathrm{CO}_{2} / \mathrm{C}_{(\mathrm{a}-\mathrm{cv})} \mathrm{O}_{2}$ ratio and arterial lactate for the whole patient population (rho $=0.64$, both $p<0.0001$ ) as well as for the subset of patients with shock (rho=0.47, $p=0.001$; and $r=0.64, p<0.0001$ respectively).

All-cause ICU mortality was $27.8 \%$. Compared to survivors, ICU non-survivors had a higher RRI [0.80 (0.10) versus $0.70(0.10), p<0.001$ ] and higher $\mathrm{P}(\mathrm{cv}-\mathrm{a}) \mathrm{CO}_{2} / \mathrm{C}(\mathrm{a}-\mathrm{cv}) \mathrm{O}_{2}$ ratio [3.67 (3.8) versus 0.91 (1.4)] and lactate levels [2.80 (2.00) versus $1.50(1.20)$ ], both $p<0.001)$. Logistic regression models showed a significant association between $\mathrm{RRI}$ and $\mathrm{P}_{(\mathrm{cv}-\mathrm{a})} \mathrm{CO}_{2} / \mathrm{C}_{(\mathrm{a}-\mathrm{cv})} \mathrm{O}_{2}$ ratio with clinical outcome. RRI showed good ability to predict ICU mortality (AUC $74.9 \%$ (95\% Cl 61\% - 88.8\%). The combination of RRI with $\mathrm{P}_{(\mathrm{cv}-}$ a) $\left.\mathrm{CO}_{2}\right) /\left(\mathrm{C}_{(\mathrm{a}-\mathrm{cv})} \mathrm{O}_{2}\right.$ ratio and lactate better predicted mortality than RRI alone [AUC 84.8\% (95\% Cl 5.1\% $94.4 \%)$ ] versus $0.74 .9 \%$, respectively, $p<0.001$ ).

Conclusions: In mechanically ventilated patients, renal blood flow impairment, assessed by the RRI on ICU admission, correlates with global tissue hypoperfusion indices. In addition, RRI in combination with tissue perfusion estimation is more valuable in predicting clinical outcome than RRI alone.

\section{Introduction}

In the context of shock pathophysiology, recent advancements in resuscitation and support of vital organs in critically ill patients include ultrasound applications aiming at the assessment of splanchnic organ perfusion [1]. Most specifically, concerning the kidney, in addition to conventional evaluation of structural abnormalities by the B-mode ultrasound, the assessment of renal blood flow by the Dopplerbased renal resistive index (RRI) measurement is currently feasible at bedside and has been considered as a tool for assessing renal perfusion in critically ill patients [2,3]. This is a simple, rapid, noninvasive 
and repeatable technique, determining the RRI by assessing the systolic and diastolic blood velocity from the Doppler flow waveforms in the intrarenal arcuate or interlobar arteries.

Originally proposed by Pourcelot as "resistive index" [4] to define the resistance of blood flow in peripheral arteries, it was later applied in intrarenal arteries, initially in patients with renal allografts, demonstrating an association between high RRI and worse outcome [5], and subsequently into a variety of renal clinical conditions [6]. Over the last decade a growing number of studies in ICU patients have documented the RRI as a promising tool for identification of patients at risk of acute kidney injury (AKI) occurrence [7, 8], AKI progression $[9,10]$ as well as for clinical outcome [11].

Initially thought to reflect intrarenal arterial resistance, the RRI is currently also considered as a reflection of renal parenchymal resistance and compliance $(8,12)$. Current data on RRI suggest a complex underlying pathophysiology, including interactions with non-renal factors such as hemodynamics [13] and peripheral circulation [3], not fully understood so far. Furthermore, there is some experimental and clinical evidence for an early intrarenal vascular response to cardiorespiratory insults, such as acute blood loss $[14,15]$ and post-cardiac surgery in the presence of low mixed venous oxygen saturation [16].

Based on the above initial evidence we hypothesized that a compromised renal blood flow, as expressed by an increased RRI on ICU admission, could be associated with global tissue hypoperfusion. Our primary objective was to evaluate the relationship between RRI and tissue hypoperfusion indices and also the clinical outcome in mechanically ventilated patients admitted to a multidisciplinary ICU. The ratio of central venous-to-arterial carbon dioxide partial pressure difference by arterial-to-central venous oxygen content difference $\left(\mathrm{P}_{(\mathrm{cv}-\mathrm{a})} \mathrm{CO}_{2} / \mathrm{C}_{(\mathrm{a}-\mathrm{cv})} \mathrm{O}_{2}\right)$ and the arterial lactate were used as indicators of the presence of global tissue hypoperfusion [17-21].

\section{Patients And Methods}

\section{Setting}

This prospective, observational study was conducted from October 2017 through September 2018 in the 25-bed, university ICU at "Evangelismos" Hospital, a tertiary-care medical center. This ICU admits critically ill medical, surgical and trauma patients. Patients with acute coronary syndromes, cardiac surgery and transplantation are managed in special units and are admitted to our ICU if they have a complicated course. The study was approved by the Hospital Ethics Committee (approval number 38/03-2017) and informed consent was obtained from all next of kin of patients.

\section{Patients and data collection}

Patients consecutively admitted to the ICU, undergoing mechanical ventilation were eligible for inclusion in the study. Exclusion criteria were the following: age $<18$ years; pregnancy; any history of chronic renal dysfunction; conditions that are known to modify RRI such as renal artery stenosis, urinary obstruction or any kidney structural damage; non-sinus cardiac rhythm; morbid obesity resulting in poor abdominal 
echogenicity; patients readmitted or patients transferred from another ICU; an expected ICU stay of less than 48 hours and lack of an arterial and/or a central venous line placed in an internal jugular or subclavian vein at the time of RRI measurement.

Demographic data, admission diagnosis, comorbidities, laboratory examinations, severity of acute illness, presence of sepsis, presence of shock, vasopressors use and dose, occurrence of AKI and ICU clinical outcome were recorded. The illness severity was evaluated by the Acute Physiology and Chronic Health Evaluation (APACHE) II [22] and the Sequential Organ Failure Assessment (SOFA) scoring systems [23], calculated on the first day of ICU admission.

\section{Protocol and Measurements}

All patients were mechanically ventilated in the assist-control mode because of acute respiratory failure of various etiologies. They had an arterial line and a central venous catheter in an internal jugular or subclavian vein inserted by the patients' attending physicians as part of the routine ICU management.

Renal ultrasonography was performed by two intensivists, experienced in this technique (GF and IP), who were not involved in patients' management, within 24 hours of ICU admission, following an initial hemodynamic stabilization. A Vivid 7 (General Electric Healthcare, UK) was used. RRI was determined as previously described [24]. Briefly, with the use of a 7.5MHz linear transducer, the investigator performed a gray-scale sonography to obtain basic anatomic information; the color Doppler was used for insonation of arcuate or interlobar arteries and the pulsed wave Doppler module was activated in order to record the velocity-time curve. RRI was determined by assessing systolic and diastolic blood velocity in the segmental arteries and applying the following formula: RRI= (peak systolic velocity minus end-diastolic velocity) / peak systolic velocity. For each kidney, three to five reproducible waveforms in three different areas of the kidney (upper, mid and lower pole) were obtained. RRI was calculated as the average of the right and the left kidney RRI values. Hemodynamic variables, including heart rate and invasive systolic and diastolic blood pressure were recorded at the time of renal ultrasonography.

Gas exchange measurements

Blood samples were drawn simultaneously from the arterial line and the central venous catheter around the time of ultrasonography, and were immediately analyzed (ABL 300, Radiometer; Copenhagen, Denmark) for determination of the following variables: partial pressure of arterial oxygen $\left(\mathrm{PaO}_{2}\right)$ and arterial carbon dioxide $\left(\mathrm{PaCO}_{2}\right)$, partial pressure of central venous oxygen $\left(\mathrm{PcvO}_{2}\right)$ and central venous carbon dioxide $\left(\mathrm{PcvCO}_{2}\right)$, hemoglobin arterial oxygen saturation $\left(\mathrm{SaO}_{2}\right)$ and central venous oxygen saturation $\left(\mathrm{ScvO}_{2}\right)$,as well as hemoglobin concentration and arterial lactate levels.

The arterial oxygen content $\left(\mathrm{CaO}_{2}\right)$ and the central venous oxygen content $\left(\mathrm{CcvO}_{2}\right)$ were calculated using the following formulas: 
$\mathrm{CaO}_{2}=\left(\mathrm{SaO}_{2} \times \mathrm{Hb} \times 1.34\right)+\left(0.0031 \times \mathrm{PaO}_{2}\right)$ and $\mathrm{CcvO}_{2}=\left(\mathrm{ScvO}_{2} \times \mathrm{Hb} \times 1.34\right)+\left(0.0031 \times \mathrm{PcvO}_{2}\right)$ respectively, as well as the difference between them (arterial-central venous oxygen content difference, $\left.\mathrm{C}_{(\mathrm{a}-\mathrm{cv})} \mathrm{O}_{2}\right)$. Then, the $\mathrm{P}_{(\mathrm{cv}-\mathrm{a})} \mathrm{CO}_{2}$ and the $\mathrm{P}_{(\mathrm{cv}-\mathrm{a})} \mathrm{CO}_{2} / \mathrm{C}_{(\mathrm{a}-\mathrm{cv})} \mathrm{O}_{2}$ ratio were calculated.

\section{Definitions}

Sepsis was defined as the combination of a known or suspected infection and acute organ dysfunction [25]. Circulatory shock was defined as hypotension (systolic blood pressure $<90 \mathrm{~mm} \mathrm{Hg}$ and/or mean arterial pressure $<65 \mathrm{mmHg}$ ), persisting despite adequate volume resuscitation, requiring administration of vasoactive agents [26]. AKI was defined by the Kidney Disease Improving Global Outcome criteria based on serum creatinine or urinary output [27]. Baseline serum creatinine was defined as the last known value measured before hospitalization. When no previous value existed, hospital admission serum creatinine value was used [8]. Occurrence of AKI was assessed within 7 days after ICU admission. An RRI value of 0.70 was considered to be the upper threshold of the normal RRI as previously proposed [1, 10 , $13,28] . \mathrm{P}_{(\mathrm{cv}-\mathrm{a})} \mathrm{CO}_{2} / \mathrm{C}_{(\mathrm{a}-\mathrm{cv})} \mathrm{O}_{2}$ ratio and arterial lactate were used as global tissue hypoperfusion indices. For the $\mathrm{P}_{(\mathrm{cv}-\mathrm{a})} \mathrm{CO}_{2} / \mathrm{C}_{(\mathrm{a}-\mathrm{c} v)} \mathrm{O}_{2}$ ratio, a value of more than 1.4 was considered abnormal, as previously proposed [17].

\section{Statistical Analysis}

Statistical data analysis was performed using the R software, version 3.6.2 (R Foundation for Statistics, Austria). Data are described as median and interquartile range (IQR) or number and percentage. In order to compare the distributions of numerical variables between two groups of patients we used the MannWhitney $U$ test due to non-normality of distributions, whereas association between qualitative factors was appropriately investigated via the chi-squared $\left(\mathrm{X}^{2}\right)$ statistic or the Fisher's exact test. Spearman correlation coefficient was used to measure the correlation between quantitative variables. Binary logistic regression models were built for the main outcomes of interest, with odds ratio (OR) and the corresponding $95 \%$ confidence interval $(\mathrm{Cl})$ reported in relation to the model covariates. The discriminative ability of a model was assessed by the area under the receiver-operating characteristic (AUROC) curve. AUROC curves were compared according to the DeLong method. The level of statistical significance was set at 0.05 .

\section{Results}

\section{Study population}

Among 562 mechanically ventilated patients admitted to the ICU during the study period, 436 patients were excluded mainly because of ICU stay of less than 48 hours $(n=113)$, history of chronic kidney disease $(n=28)$, no sinus cardiac rhythm $(n=22)$, lack of an internal jugular or subclavian vein $(n=168)$, obesity $(n=14)$, transferred from another ICU, $(n=18)$ or ICU readmission $(n=24)$ and unavailability of investigators who performed RRI $(n=49)$. Finally, 126 mechanically ventilated patients [61 (28) years, $74 \%$ males] were included. Sepsis was present in 57 (45.2\%) patients. Sixty patients suffered from circulatory shock; in 58 (96\%) of these patients norepinephrine was administered at a dose > 
$0.1 \mu \mathrm{g} / \mathrm{kg} / \mathrm{min}$. No other vasoconstrictor agent was administered. All-cause ICU mortality was $27 \%$ $(35 / 126)$.

Seventy-seven patients had RRI >0.7. Demographic and clinical characteristics of all patients on ICU admission grouped on the basis of this cutoff, are shown in Table 1. Patients with RRI >0.7, compared to those with RRI $\leq 0.7$, were older and more severely ill, they had more often history of arterial hypertension, presence of sepsis and shock, lower diastolic and mean arterial pressure, and higher values of tissue oxygenation indices. The occurrence of AKI was $42.9 \%$ (54/126). Median RRI value was $0.82(0.07)$ in patients who developed AKI and $0.68(0.1)$ in patients who did not, $p<0.001)$. 
Table 1

Demographic, clinical and laboratory characteristics of study patients on ICU admission and differences between patients with $\mathrm{RRI} \leq 0.7$ and patients with $\mathrm{RRI}>0.7^{a}$

\begin{tabular}{|c|c|c|c|c|}
\hline variables & $\begin{array}{l}\text { All } \\
\text { patients } \\
n=126\end{array}$ & $\begin{array}{l}\text { Patients with RRI } \\
\leq 0.7 \\
\mathrm{n}=49\end{array}$ & $\begin{array}{l}\text { Patients with RRI } \\
>0.7 \\
\mathrm{n}=77\end{array}$ & $\begin{array}{l}p \\
\text { value }\end{array}$ \\
\hline Age, years, & $61(27.8)$ & $46(28)$ & $70(22)$ & $\begin{array}{l}<.001 \\
0.0\end{array}$ \\
\hline Sex, male, n (\%) & $74(58.7)$ & $30(61.2)$ & $44(57.1)$ & 0.78 \\
\hline $\begin{array}{l}\text { Illness severity scores at ICU } \\
\text { admission }\end{array}$ & $9(3)$ & $8(3)$ & $9(3)$ & $\hat{0} .001$ \\
\hline $\begin{array}{l}\text { Admission diagnosis } \\
\text { Medical } \\
\text { Surgical } \\
\text { Trauma non- surgical }\end{array}$ & $\begin{array}{l}46(36.5) \\
61(48.4) \\
19(15.1)\end{array}$ & $\begin{array}{l}17(34.7) \\
20(40.8) \\
12(24.5)\end{array}$ & $\begin{array}{l}29(37.7) \\
41(53.2) \\
7(9.1)\end{array}$ & 0.05 \\
\hline AKI occurrence, $n(\%)$ & $54(42.9)$ & $2(4.1)$ & $52(67.5)$ & $\hat{0} .001$ \\
\hline $\begin{array}{l}\text { History of arterial hypertension, } \mathrm{n} \\
(\%)\end{array}$ & $30(23.8)$ & $7(14.3)$ & 23 (29.9) & $\begin{array}{l}<.001 \\
0.07\end{array}$ \\
\hline \multicolumn{5}{|l|}{ Characteristics at inclusion } \\
\hline Presence of sepsis, n (\%) & $57(45.2)$ & $14(28.6)$ & $43(55.8)$ & 0.004 \\
\hline Presence of shock, n (\%) & $60(47.6)$ & $7(14.3)$ & $53(68.8)$ & $\begin{array}{l}<.001 \\
0.00\end{array}$ \\
\hline $\begin{array}{l}\text { Norepinephrine }>0.1 \mu \mathrm{g} / \mathrm{kg} / \mathrm{min}, \mathrm{n} \\
(\%)\end{array}$ & $58(46)$ & $4(8.2)$ & $54(70.1)$ & $\hat{0.001}$ \\
\hline Systolic arterial pressure, $\mathrm{mmHg}$ & $\begin{array}{l}132 \\
(25.8)\end{array}$ & $132(21)$ & $132(28)$ & 0.28 \\
\hline \multicolumn{5}{|c|}{$\begin{array}{l}\text { apresented as median (IQR); ICU, intensive care unit; } \mathrm{AKI} \text {, acute kidney injury; } \mathrm{APACHE} \text {, Acute } \\
\text { Physiology and Chronic Health Evaluation; } \mathrm{SOFA} \text {, Sequential Organ Failure Assessment; FiO2 PaO2, } \\
\text { partial pressure of arterial oxygen; } \mathrm{PCO}_{2} \text {, partial pressure of arterial carbon dioxide } \mathrm{SaO}_{2} \text {, hemoglobin } \\
\text { arterial oxygen saturation; } \mathrm{PcvO}_{2}, \text { partial pressure of central venous oxygen; } \mathrm{PcvCO}_{2} \text { partial pressure } \\
\text { of central venous carbon dioxide; } \mathrm{ScvO}_{2} \text {, central venous oxygen saturation; } \mathrm{CaO}_{2} \text {, arterial oxygen } \\
\text { content } \mathrm{C}_{\mathrm{cv}} \mathrm{O}_{2} \text { central venous oxygen content } \mathrm{P}(\mathrm{cv}-\mathrm{a}) \mathrm{CO}_{2} \text { central venous-arterial carbon dioxide tension } \\
\text { difference; } \mathrm{C}_{(\mathrm{a}-\mathrm{cv})} \mathrm{O}_{2} \text {, arterio-central venous oxygen content difference; RRI, renal resistive index }\end{array}$} \\
\hline
\end{tabular}




\begin{tabular}{|c|c|c|c|c|}
\hline \multirow[t]{2}{*}{ variables } & $\begin{array}{l}\text { All } \\
\text { patients }\end{array}$ & $\begin{array}{l}\text { Patients with RRI } \\
\leq 0.7\end{array}$ & $\begin{array}{l}\text { Patients with RRI } \\
>0.7\end{array}$ & \multirow[t]{2}{*}{$\begin{array}{l}p \\
\text { value }\end{array}$} \\
\hline & $n=126$ & $n=49$ & $\mathrm{n}=77$ & \\
\hline Diastolic arterial pressure, $\mathrm{mmHg}$ & $65(15)$ & $71(16)$ & $64(15)$ & 0.01 \\
\hline Mean arterial pressure, $\mathrm{mmHg}$ & $86(17.8)$ & $89(12)$ & $83(17)$ & 0.03 \\
\hline Heart rate, beats /min & $86.5(27)$ & $89(28)$ & $86(28)$ & 0.72 \\
\hline Arterial lactate, mmol/l & $1.8(1.8)$ & $1.2(0.6)$ & $2.4(2.2)$ & $\begin{array}{l}< \\
0.001\end{array}$ \\
\hline Serum creatinine, mg/dl & $1(0.87)$ & $0.8(0.4)$ & $1.1(1.1)$ & $\begin{array}{l}< \\
0.001\end{array}$ \\
\hline Hemoglobin, g/dl & $\begin{array}{l}10.35 \\
(2.8)\end{array}$ & $10.6(2.5)$ & $10.1(2.8)$ & 0.09 \\
\hline RRI & $0.74(0.1)$ & $0.64(0.06)$ & $0.79(0.08)$ & $\begin{array}{l}<.001 \\
0.001\end{array}$ \\
\hline \multicolumn{5}{|l|}{ Gas exchange } \\
\hline $\mathrm{FiO}_{2}$ & $0.6(0.1)$ & $0.6(0.15)$ & $0.6(0.1)$ & 0.80 \\
\hline $\mathrm{PaO}_{2}, \mathrm{mmHg}$ & $\begin{array}{l}116.6 \\
(59.7)\end{array}$ & $115.3(64.3)$ & $117.2(57.7)$ & 0.64 \\
\hline $\mathrm{PCO}_{2}, \mathrm{mmHg}$ & $\begin{array}{l}38.65 \\
(7.2)\end{array}$ & 39.2 (5.9) & $38.2(8)$ & 0.16 \\
\hline $\mathrm{pH}$ & $7.38(0.1)$ & $7.38(0.08)$ & $7.38(0.08)$ & 0.06 \\
\hline $\mathrm{SaO}_{2}, \%$ & $\begin{array}{l}98.05 \\
(1.9)\end{array}$ & $98.1(2.1)$ & $98(1.8)$ & 0.86 \\
\hline $\mathrm{PcvO}_{2}, \mathrm{mmHg}$ & $45.5(9.0)$ & $46.6(8.9)$ & $44.5(9.2)$ & 0.15 \\
\hline $\mathrm{PcvCO}_{2}, \mathrm{mmHg}$ & $\begin{array}{l}44.5 \\
(10.7)\end{array}$ & 41.4 (8.2) & $47(9.4)$ & $\hat{0.001}$ \\
\hline $\mathrm{ScvO}_{2}, \%$ & $78.9(9.7)$ & $79(9.4)$ & 78.8 (9.7) & 0.82 \\
\hline $\mathrm{CaO}_{2}, \mathrm{ml} / \mathrm{dl}$ & $\begin{array}{l}13.98 \\
(3.5)\end{array}$ & 14.54 (2.75) & 13.51 (3.49) & 0.06 \\
\hline \multicolumn{5}{|c|}{ 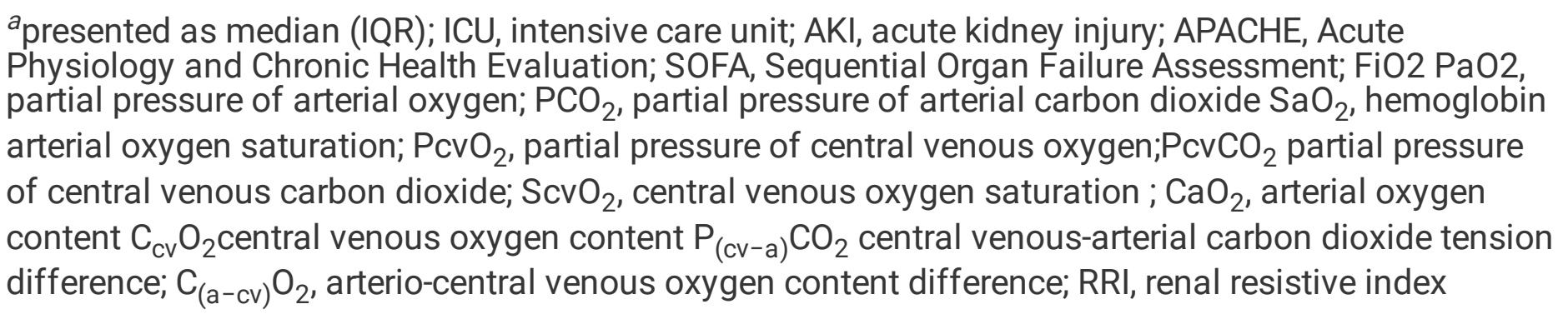 } \\
\hline
\end{tabular}




\begin{tabular}{|c|c|c|c|c|}
\hline \multirow[t]{2}{*}{ variables } & $\begin{array}{l}\text { All } \\
\text { patients }\end{array}$ & $\begin{array}{l}\text { Patients with RRI } \\
\leq 0.7\end{array}$ & $\begin{array}{l}\text { Patients with RRI } \\
>0.7\end{array}$ & \multirow[t]{2}{*}{$\begin{array}{l}p \\
\text { value }\end{array}$} \\
\hline & $n=126$ & $n=49$ & $\mathrm{n}=77$ & \\
\hline $\mathrm{C}_{\mathrm{cv}} \mathrm{O}_{2}, \mathrm{ml} / \mathrm{dl}$ & $\begin{array}{l}10.78 \\
(2.7)\end{array}$ & $11.22(2.65)$ & $10.29(2.82)$ & 0.08 \\
\hline $\mathrm{P}_{(\mathrm{cv}-\mathrm{a})} \mathrm{CO}_{2}, \mathrm{mmHg}$ & $3.6(8.4)$ & $1.9(1.4)$ & $8.7(9.2)$ & $<.001$ \\
\hline $\mathrm{C}_{(\mathrm{a}-\mathrm{cv})} \mathrm{O}_{2}, \mathrm{ml} / \mathrm{dl}$ & $2.88(1.6)$ & $2.88(1.6)$ & $2.87(1.6)$ & 0.57 \\
\hline $\begin{array}{l}\mathrm{P}_{(\mathrm{cv}-\mathrm{a})} \mathrm{CO}_{2} / \mathrm{C}_{(\mathrm{a}-\mathrm{cv})} \mathrm{O}_{2}, \mathrm{mmHg} / \mathrm{ml} \\
\mathrm{O} 2\end{array}$ & $\begin{array}{l}1.32 \\
(2.60)\end{array}$ & $0.62(0.57)$ & $2.88(3.39)$ & $\hat{0.001}$ \\
\hline \multicolumn{5}{|l|}{ Outcome } \\
\hline Length of ICU stay (days) & $14(17)$ & $10(12)$ & $15(17)$ & 0.001 \\
\hline ICU mortality, n (\%) & $35(27.8)$ & $1(2)$ & $34(44.2)$ & $\dot{\delta} 001$ \\
\hline \multicolumn{5}{|c|}{$\begin{array}{l}\text { apresented as median (IQR); ICU, intensive care unit; AKI, acute kidney injury; } \mathrm{APACHE} \text {, Acute } \\
\text { Physiology and Chronic Health Evaluation; } \mathrm{SOFA} \text {, Sequential Organ Failure Assessment; FiO2 PaO2, } \\
\text { partial pressure of arterial oxygen; } \mathrm{PCO}_{2} \text {, partial pressure of arterial carbon dioxide } \mathrm{SaO}_{2} \text {, hemoglobin } \\
\text { arterial oxygen saturation; } \mathrm{PcvO}_{2} \text {, partial pressure of central venous oxygen; } \mathrm{PcvCO}_{2} \text { partial pressure } \\
\text { of central venous carbon dioxide; } \mathrm{ScvO}_{2} \text {, central venous oxygen saturation; } \mathrm{CaO}_{2} \text {, arterial oxygen } \\
\text { content } \mathrm{C}_{\mathrm{cv}} \mathrm{O}_{2} \text { central venous oxygen content } \mathrm{P}\left(\mathrm{cv}-\mathrm{a} \text { ) } \mathrm{CO}_{2} \text { central venous-arterial carbon dioxide tension }\right. \\
\text { difference; } \mathrm{C}_{(\mathrm{a}-\mathrm{cv})} \mathrm{O}_{2} \text {, arterio-central venous oxygen content difference; RRI, renal resistive index }\end{array}$} \\
\hline
\end{tabular}

\section{RRI and the presence of circulatory shock}

Patients with shock had a significantly higher RRI than patients without shock [0.80 (0.10) versus 0.68 (0.10) respectively, $p<0.001]$. Similarly, compared to patients under norepinephrine dose $\leq 0.1$ $\mu \mathrm{g} / \mathrm{kg} / \mathrm{min}$, patients under norepinephrine dose $>0.1 \mu \mathrm{g} / \mathrm{kg} / \mathrm{min}$ had significantly higher RRI values [80 $(0.1)$ versus $0.68(0.1)$, respectively, $p<0.001]$.

Both RRI and $\mathrm{P}_{(\mathrm{cv}-\mathrm{a})} \mathrm{CO}_{2} / \mathrm{C}_{(\mathrm{a}-\mathrm{cv})} \mathrm{O}_{2}$ ratio were significantly associated with shock in univariate regression analyses (both $p$-values $<0.001$ ). The odds of shock for a patient with RRI $>0.7$ were 13.2 times the odds of shock for a patient with $\mathrm{RRI} \leq 0.7$, Table 2 . When both variables were inserted as covariates in a multivariate logistic regression model, their statistical significance was maintained. When lactate was inserted into the model (on the grounds of being significantly correlated with shock when univariately analyzed ( $p$-value $<0.0001), \mathrm{P}_{(\mathrm{cv}-\mathrm{a})} \mathrm{CO}_{2} / \mathrm{C}_{(\mathrm{a}-\mathrm{cv})} \mathrm{O}_{2}$ ratio maintained its statistical significance whereas RRI did not, Table 2. 
Table 2

Logistic regression models for detecting shock. Odds ratios (OR) with 95\% confidence intervals (95\% Cl) and the covariate $\mathrm{p}$-value are reported

\begin{tabular}{|lccc|}
\hline Model / Variables & OR & $95 \% \mathrm{Cl}$ & P-value \\
\hline Model 1 & & & \\
$\mathrm{RRI}>0.7$ & 13.2 & $5.2-33.7$ & $<0.001$ \\
\hline Model 2 & & & \\
$\left.\mathrm{P}_{(\mathrm{cv}-\mathrm{a})} \mathrm{CO}_{2}\right) /\left(\mathrm{C}_{(\mathrm{a}-\mathrm{cv})} \mathrm{O}_{2}>1.4\right.$ & 25.0 & $9.8-63.0$ & $<0.001$ \\
\hline Model 3 & & & \\
\hline $\mathrm{RRI}>0.7$ & 3.8 & $1.1-13.2$ & 0.038 \\
\hline $\left.\mathrm{P}_{(\mathrm{cv}-\mathrm{a})} \mathrm{CO}_{2}\right) /\left(\mathrm{C}_{(\mathrm{a}-\mathrm{cv})} \mathrm{O}_{2}>1.4\right.$ & 15.0 & $5.2-42.9$ & $<0.001$ \\
\hline Model 4 & & & \\
\hline Lactate $>2$ mmol/l & 8.7 & $2.8-27.1$ & 0.0002 \\
\hline $\mathrm{RRI}>0.7$ & 1.8 & $0.5-6.4$ & 0.35 \\
\hline $\left.\mathrm{P}_{(\mathrm{cv}-\mathrm{a})} \mathrm{CO}_{2}\right) /\left(\mathrm{C}_{(\mathrm{a}-\mathrm{cv})} \mathrm{O}_{2}>1.4\right.$ & 10.1 & $3.3-30.6$ & $<0.001$ \\
\hline $\begin{array}{l}\left.\mathrm{RRI}, \text { renal resistive index; } \mathrm{P}_{(\mathrm{cv}-\mathrm{a})} \mathrm{CO}_{2}\right) / \mathrm{C}_{(\mathrm{a}-\mathrm{cv})} \mathrm{O}_{2} \text {, ratio of central venous-to-arterial carbon dioxide } \\
\text { partial pressure difference by arterial-to-central venous oxygen content difference }\end{array}$ & \\
\hline
\end{tabular}

\section{Relation between RRI and tissue hypoperfusion indices}

Tissue hypoperfusion indices i. e., arterial lactate and $\mathrm{P}_{(\mathrm{cv}-\mathrm{a})} \mathrm{CO}_{2} / \mathrm{C}_{(\mathrm{a}-\mathrm{cv})} \mathrm{O}_{2}$ ratio were significantly higher in patients with RRI $>0.7$ compared to those with $\mathrm{RRI} \leq 0.7$, Table 1 . Taking into account the whole study population there was a linear correlation between RRI and $\mathrm{P}_{(\mathrm{cv}-\mathrm{a})} \mathrm{CO}_{2} / \mathrm{C}_{(\mathrm{a}-\mathrm{cv})} \mathrm{O}_{2}$ ratio, as well as between $\mathrm{RRI}$ and arterial lactate (Spearmans' rho $=0.64, \mathrm{p}<0.0001$ in both cases, Figs. $1 \mathrm{a}$ and $1 \mathrm{~b}$ ). Analysis in the subset of patients with shock $(n=60)$ exhibited similar results: rho $=0.47, p=0.001$; and $r=0.64, p<$ 0.0001 respectively, Figs. $1 \mathrm{c}$ and $1 \mathrm{~d}$.

\section{RRI, hypoperfusion indices and clinical outcome}

All-cause ICU mortality was $27.8 \%$., Table 1 . Median RRI value on ICU admission was 0.7 (0.1) in survivors and 0.8 (0.1) in non-survivors, $\mathrm{p}<0.001$. Also, compared to survivors, ICU non-survivors had a higher $\mathrm{P}$ (cva) $\mathrm{CO}_{2} / \mathrm{C}(\mathrm{a}-\mathrm{cv}) \mathrm{O}_{2}$ ratio [3.67 (3.8) versus 0.91 (1.4)] and higher lactate levels [2.80 (2.00) versus 1.50 (1.20)], both $p<0.001)$. Univariate logistic regression models showed a significant association between $\mathrm{RRI}$ and $\mathrm{P}_{(\mathrm{cv}-\mathrm{a})} \mathrm{CO}_{2} / \mathrm{C}_{(\mathrm{a}-\mathrm{cv})} \mathrm{O}_{2}$ ratio with clinical outcome, Table 3, Model 1 and Model 2. When all variables were used as covariates in a multivariate logistic regression model, the statistical significance for RRI and $\left.\mathrm{P}(\mathrm{cv}-\mathrm{a}) \mathrm{CO}_{2}\right) /\left(\mathrm{C}(\mathrm{a}-\mathrm{cv}) \mathrm{O}_{2}\right.$ ratio was maintained, Table 3. Of note, $\mathrm{P}(\mathrm{cv}-\mathrm{a}) \mathrm{CO}_{2}$ was not inserted in the 
models where $\left.\mathrm{P}(\mathrm{cv}-\mathrm{a}) \mathrm{CO}_{2}\right) /\left(\mathrm{C}(\mathrm{a}-\mathrm{cv}) \mathrm{O}_{2}\right.$ ratio was also present, in order to avoid multicollinearity issues which may mask the impact of each of those covariates on the dependent variable (rho $=93 \%$ ).

Table 3

Logistic regression models for predicting the probability of death. Odds ratios $(\mathrm{OR})$ with $95 \%$ confidence intervals $(95 \% \mathrm{Cl})$ and the covariate $\mathrm{p}$-value are reported.

\begin{tabular}{|c|c|c|c|}
\hline Model / Variables & OR & $95 \% \mathrm{Cl}$ & p-value \\
\hline \multicolumn{4}{|l|}{ Model 1} \\
\hline $\mathrm{RRI}>0.7$ & 37.9 & $4.9-289.1$ & $<0.001$ \\
\hline \multicolumn{4}{|l|}{ Model 2} \\
\hline $\left.\mathrm{P}_{(\mathrm{cv}-\mathrm{a})} \mathrm{CO}_{2}\right) /\left(\mathrm{C}_{(\mathrm{a}-\mathrm{cv})} \mathrm{O}_{2}>1.4\right.$ & 15.7 & $5.1-48.7$ & $<0.001$ \\
\hline \multicolumn{4}{|l|}{ Model 3} \\
\hline $\mathrm{RRI}>0.7$ & 14.8 & $1.8-122.3$ & 0.005 \\
\hline $\left.\mathrm{P}_{(\mathrm{cv}-\mathrm{a})} \mathrm{CO}_{2}\right) /\left(\mathrm{C}_{(\mathrm{a}-\mathrm{cv})} \mathrm{O}_{2}>1.4\right.$ & 6.7 & $2.0-22.3$ & 0.001 \\
\hline \multicolumn{4}{|l|}{ Model 4} \\
\hline $\mathrm{RR} \mid>0.7$ & 10.0 & $1.1-89.0$ & 0.038 \\
\hline $\left.\mathrm{P}_{(\mathrm{cv}-\mathrm{a})} \mathrm{CO}_{2}\right) /\left(\mathrm{C}_{(\mathrm{a}-\mathrm{cv})} \mathrm{O}_{2}>1.4\right.$ & 5.0 & $1.4-17.8$ & 0.013 \\
\hline Lactate $>2 \mathrm{mmol} / \mathrm{l}$ & 2.1 & $0.7-6.5$ & 0.21 \\
\hline APACHE II score & 1.0 & $1.0-1.1$ & 0.42 \\
\hline
\end{tabular}

RRI, renal resistive index; $\left.\mathrm{P}_{(\mathrm{cv}-\mathrm{a})} \mathrm{CO}_{2}\right) / \mathrm{C}_{(\mathrm{a}-\mathrm{cv})} \mathrm{O}_{2}$, ratio of central venous-to-arterial carbon dioxide partial pressure difference by arterial-to-central venous oxygen content difference $\mathrm{ROC}$ analyses estimating the predictive performance of $\mathrm{RRI}, \mathrm{P}_{(\mathrm{cv}-\mathrm{a})} \mathrm{CO}_{2} / \mathrm{C}_{(\mathrm{a}-\mathrm{cv})} \mathrm{O}_{2}$ ratio, arterial lactate and their combination with regard to ICU mortality are presented in Fig. 2. The combination of RRI with $\left.\mathrm{P}_{(\mathrm{cv}-\mathrm{a})} \mathrm{CO}_{2}\right) /\left(\mathrm{C}_{(\mathrm{a}-\mathrm{cv})} \mathrm{O}_{2}\right.$ ratio and lactate predicted better the clinical outcome than each one index alone: AUC ROC of the combined model was $84.8 \%$ versus $74.9 \%$ for RRI alone, $p=0.0001$; versus $77.8 \%$ for $\left.\mathrm{P}_{(\mathrm{cv}-\mathrm{a})} \mathrm{CO}_{2}\right) /\left(\mathrm{C}_{(\mathrm{a}-\mathrm{cv})} \mathrm{O}_{2}\right.$ ratio alone, $\mathrm{p}<0.002$; versus $74.6 \%$ for lactate alone, $\mathrm{p}=0.004$, DeLong tests.

\section{Discussion}

The main findings of this prospective observational study are the following: RRI value on first day of ICU admission (i) significantly correlates with $\left.\mathrm{P}_{(\mathrm{cv}-\mathrm{a})} \mathrm{CO}_{2}\right) /\left(\mathrm{C}_{(\mathrm{a}-\mathrm{cv})} \mathrm{O}_{2}\right)$ ratio and arterial lactate levels; ii) is 
independently associated with the presence of circulatory shock; (iii) its combination with $\left.\mathrm{P}_{(\mathrm{cv}-\mathrm{a})} \mathrm{CO}_{2}\right)$ / $\left(\mathrm{C}_{(\mathrm{a}-\mathrm{cv})} \mathrm{O}_{2}\right.$ ratio and arterial lactate better predicts the ICU mortality than each one index separately.

The finding of the significant association between RRI and global tissue hypoperfusion indices, to the best of our knowledge, is first described in the present study. This finding supports the hypothesis that under conditions of tissue hypoperfusion, the renal vasculature can early respond to an oxygen supply and demand mismatch by modulating the vascular tone. Furthermore, it is indirectly consistent with the findings of a recent study demonstrating that RRI of patients admitted with shock was higher than RRI in patients without shock [13]. Likewise, a similar relationship between RRI and shock was also observed in the present study. The odds of shock for a patient with abnormal RRI were 13.2 times the odds of shock for a patient with normal RRI. Therefore, by extrapolating this finding, the association we found between $\mathrm{RRI}$ and tissue oxygenation indices seems to be expected and reasonable.

The ability of RRI to detect, beyond a renal blood flow impairment, global tissue hypoperfusion provides evidence to the recently reported concept of the ultrasound-based assessment of visceral end-organ perfusion in the critically ill [1], expanding thus, the bedside clinical monitoring window for hypoperfusion in shock states and offering new insights in shock pathophysiology. Corradi et al, have provided analogous evidence in normotensive polytrauma patients without biochemical signs of hypoperfusion [15]. Specifically, they reported that a RRI greater than 0.7 on admission to the emergency department was predictive of progression to hemorrhagic shock suggesting thus, that renal blood flow redistribution occurs very early in response to an occult bleeding. Similarly, early detection of peripheral hypoperfusion through the evaluation of RRI has recently been demonstrated in a patient with occult blood loss, enhancing thus, the role of RRI as a new index in critical care [29]. Furthermore, in another study in patients with acute lung injury, short-term mild hypoxemia has been reported to increase RRI (30).

Interestingly, in accordance with our findings, a positive association of RRI and lactate has also been described in a recent study dealing with factors associated with RRI in critically ill patients [28]. However, in that study the interpretation of this association, by the authors, was that RRI values might be associated with greater clinical severity, because lactate had been considered as an isolated severity marker.

Taken together, the present data, in conjunction with findings from the aforementioned studies, underline the importance of RRI as a potential surrogate marker for assessment of systemic hypoperfusion, besides the other hemodynamic parameters routinely used in daily practice. Of note, since hemodynamic disturbance could influence RRI, we performed the RRI assessment within the first 24 hours, after a seeming normalization of the systemic blood pressure. Therefore, despite a restored arterial pressure, an increased RRI value could unmask a persistence of tissue hypoperfusion, warning thus, for closer and probably further titration of the hemodynamic treatment.

Among all the $\mathrm{O}_{2}$ - and $\mathrm{CO}_{2}$-derived parameters, the venous-arterial content $\mathrm{CO}_{2}\left(\mathrm{C}_{\mathrm{v}-\mathrm{a}} \mathrm{CO}_{2}\right)$ to $\mathrm{C}_{(\mathrm{a}-\mathrm{v})} \mathrm{O}_{2}$ is considered a reliable marker of global anaerobic metabolism (17-21). Because the calculation of 
$\mathrm{P}_{(\mathrm{v}-\mathrm{a})} \mathrm{CO}_{2}$ is simpler, $\mathrm{P}_{(\mathrm{v}-\mathrm{a})} \mathrm{CO}_{2}$ has been used to replace $\mathrm{C}_{(\mathrm{v}-\mathrm{a})} \mathrm{CO}_{2}$ in the clinical practice. Although it could be argued that there may be a difference between the $\mathrm{PCO}_{2}$ and the $\mathrm{CCO}_{2}$, the relation between them is almost linear over the physiological range [19]. Furthermore, $\mathrm{P}_{(\mathrm{v}-\mathrm{a})} \mathrm{CO}_{2} / \mathrm{C}_{(\mathrm{a}-\mathrm{v})} \mathrm{O}_{2}$ ratio highly correlates with the arterial lactate levels $[17,31]$. In the analyses, we used the threshold value of 1.4 of this ratio, since Mekontso-Dessap et al, have shown that it better predicts the presence of hyperlactatemia, compared to other parameters [17]. Since pulmonary artery catheters are less commonly used today than in the past [32], we sampled the central venous blood, as a surrogate of mixed venous blood for the $\mathrm{P}_{(\mathrm{v}-\mathrm{a})} \mathrm{CO}_{2} / \mathrm{C}_{(\mathrm{a}-\mathrm{v})} \mathrm{O}_{2}$ ratio calculation as previously reported [20].

Clinical outcome in ICU patients is known to be complex and multifactorial. In the present study, we found an association of RRI with ICU mortality; this finding is in agreement with that of a previous study in critically ill patients [11]. Interestingly, we further found that $\left.\mathrm{P}_{(\mathrm{v}-\mathrm{a})} \mathrm{CO}_{2}\right) /\left(\mathrm{C}_{(\mathrm{a}-\mathrm{v})} \mathrm{O}_{2}\right.$ and lactate were also related to mortality and that the combination of $\mathrm{RRI}$, lactate and $\left.\mathrm{P}_{(\mathrm{v}-\mathrm{a})} \mathrm{CO}_{2}\right) /\left(\mathrm{C}_{(\mathrm{a}-\mathrm{v})} \mathrm{O}_{2}\right)$ ratio further improves the prognostic accuracy. To interpret this novel finding we must take into account that the $\left.\mathrm{P}_{(\mathrm{v}-\mathrm{a})} \mathrm{CO}_{2}\right) /\left(\mathrm{C}_{(\mathrm{a}-\mathrm{v})} \mathrm{O}_{2}\right)$ ratio expresses the global hypoperfusion, whereas an increased RRI expresses endorgan hypoperfusion of a vital organ (kidney), whose dysfunction significantly increases the mortality risk [33-35]. Therefore, this could be a probable explanation for the better performance of their combination in outcome prediction, and underlines the need for evaluation of blood flow in vital endorgans, in addition to global tissue hypoperfusion indices, in order to guide therapies to reduce mortality.

Certain limitations of the present study should be pointed out. Firstly, the non-homogeneous sample of critically ill patients suffering from various types of shock did not allow for separate information on different tissue hypoxia types. Secondly, the specific role of large-vessel dysfunction, such as arterial stiffness parameters [36] on RRI value was not assessed. Nevertheless, the data hereby presented, showing the significant correlation between RRI and tissue hypoperfusion indices in a mixed ICU population, might indicate a further role of RRI in this context.

\section{Conclusion}

In summary, we have shown that RRI is a marker that serves, beyond its known role in evaluation of renal blood flow, as a surrogate marker of global tissue perfusion. In addition, its combination with $\mathrm{P}_{(\mathrm{cv}-\mathrm{a})} \mathrm{CO}_{2} /$ $\mathrm{C}_{(\mathrm{a}-\mathrm{cv})} \mathrm{O}_{2}$ ratio and lactate is more valuable in predicting clinical outcome than each one index separately. Therefore, RRI could provide a potential of warning for additional monitoring need and for guiding the clinical management aiming at a better outcome of critically ill patients. More studies further investigating the role of RRI in ICU are worthwhile.

\section{Abbreviations}

ICU

Intensive care unit 
RRI

renal resistive index

AKI

acute kidney injury

$\mathrm{PaO}_{2}$

partial pressure of arterial oxygen

$\mathrm{PaCO}_{2}$

partial pressure arterial carbon dioxide

$\mathrm{PCvO}_{2}$

partial pressure of central venous oxygen

$\mathrm{PcvCO}_{2}$

partial pressure of central venous carbon dioxide

$\mathrm{SaO}_{2}$

hemoglobin arterial oxygen saturation

$\mathrm{ScvO}_{2}$

hemoglobin central venous oxygen saturation

\section{Declarations}

- Ethics approval and consent to participate: The study was approved by the Hospital Ethics Committee (approval number 38/03-2017) and informed consent was obtained from all next of kin of patients.

- Consent for publication: The manuscript has been read and approved for submission and publication by all authors.

- Availability of data and materials: The datasets used and analyzed during the current study are available from the corresponding author on reasonable request.

- Competing interests: None

- Funding : No

- Authors' contributions: All authors contributed to this work. IP, CR and SZ conceived and designed the study. GF and IP performed the renal ultrasounds. EC and SK performed statistical analysis. The first draft was written by GF, SK, SZ and CR. IB, EB and AB contributed to data interpretation and critical revision of the manuscript. All authors read and approved the final manuscript.

- Acknowledgements: Not applicable

\section{References}

1. Corradi F, Via G, Tavazzi G. What's new in ultrasound-based assessment of organ perfusion in the critically ill: expanding the bedside clinical monitoring window for hypoperfusion in shock. Intensive Care Med. 2020;46:775-9. 
2. Faubel S, Patel NU, Lockhart ME, Cadnapaphornchai MA. Renal relevant radiology: use of ultrasonography in patients with AKI. Clin J Am SocNephrol. 2014;9:382-94.

3. Cauwenberghs N, Kuznetsova T. Determinants and Prognostic Significance of the Renal Resistive Index. Pulse. 2015;3:172-8.

4. Pourcelot L. Applications cliniques de le'xamen Doppler examinations transcutane. In: Peronneau $P$, editor. Velocimetrie Ultrasonore Doppler. Paris: Inserm; 1971. pp. 213-7.

5. Radermacher J, Mengel M, Ellis S, Stuht S, Hiss M, Schwarz A, et al. The renal arterial resistance index and renal allograft survival. N Engl J Med. 2003;349:115-24.

6. Di Nicolò P, Granata A. Renal intraparenchymal resistive index: the ultrasonographic answer to many clinical questions. Journal of Nephrology. 2019;32:527-38.

7. Schnell D, Deruddre S, Harrois A, Pottecher J, Cosson C, Adoui N, et al. Renal resistive index better predicts the occurrence of acute kidney injury than cystatin C. Shock. 2012;38:592-7.

8. Haitsma Mulier JLG, Rozemeijer S, Rottgering JG, Spoelstra-de Man AME, Elbers PWG, Tuinman PR, et al. Renal resistive index as an early predictor and discriminator of acute kidney injury in critically ill patients; a prospective observational cohort study. PLoS ONE. 2018;13:e0197967.

9. Lerolle N, Guérot E, Faisy C, Bornstain C, Diehl J-L, Fagon J-Y. Renal failure in septic shock: predictive value of Doppler-based renal arterial resistive index. Intensive Care Med. 2006;32:1553-9.

10. Darmon M, Schortgen F, Vargas F, Liazydi A, Schlemmer B, Brun-Buisson C, et al. Diagnostic accuracy of Doppler renal resistive index for reversibility of acute kidney injury in critically ill patients. Intensive Care Med. 2011;37:68-76.

11. Boddi M, Bonizzoli M, Chiostri M, Begliomini D, Molinaro A, TadiniBuoninsegni L, Gensini GF, Peris A. Renal Resistive Index and mortality in critical patients with acute kidney injury. Eur $\mathrm{J}$ Clin Invest. 2016;46:242-51.

12. Tublin ME, Bude RO, Platt JF. RRI in renal Doppler sonography: where do we stand? AJR 2003;180:885 - 92.

13. Rozemeijer S, HaitsmaMulier JLG, Röttgering JG, Elbers PWG, Spoelstra-de Man AME, Tuinman PR, et al. Renal Resistive Index: Response to Shock and its Determinants in Critically III Patients. Shock. 2019;52:43-51.

14. Whitehouse T, Stotz M, Taylor V, Stidwill R, Singer M. Tissue oxygen and hemodynamics in renal medulla, cortex, and corticomedullary junction during hemorrhage-reperfusion. Am J Physiol Renal Physiol. 2006;291:F647-53.

15. Corradi F, Brusasco C, Vezzani A, Palermo S, Altomonte F, Moscatelli P, Pelosi P. Hemorrhagic shock in polytrauma patients: early detection with renal Doppler resistive index measurements. Radiology. 2011;260:112-8.

16. Corradi F, Brusasco C, Paparo F, Manca T, Santori G, Benassi F, et al. Renal Doppler resistive index as a marker of oxygen supply and demand mismatch in postoperative cardiac surgery patients. Biomed Res Int. 2015; Article ID 763940. 
17. Mekontso-Dessap A, Castelain V, Anguel N, Bahloul M, Schauvliege F, Richard C, Teboul JL. Combination of venoarterial $\mathrm{PCO}_{2}$ difference with arteriovenous $\mathrm{O}_{2}$ content difference to detect anaerobic metabolism in patients. Intensive Care Med. 2002;28:272-7.

18. Ospina-Tascón GA, Umaña M, Bermúdez W, Bautista-Rincon DF, Hernández G, Bruhn A, et al. Combination of arterial lactate levels and venous-arterial $\mathrm{CO}_{2}$ to arterial-venous $\mathrm{O}_{2}$ content difference ratio as markers of resuscitation in patients with septic shock. Intensive Care Med. 2015;41:796805.

19. OspinaTascón GA, Hernández G, Cecconi M. Understanding the venous-arterial $\mathrm{CO}_{2}$ to arterialvenous $\mathrm{O}_{2}$ content difference ratio. Intensive Care Med. 2016;42:1801-4.

20. Mesquida J, Saludes P, Gruartmoner G, Espinal C, Torrents E, Baigorri F, Artigas A. Central venous-toarterial carbon dioxide difference combined with arterial-to-venous oxygen content difference is associated with lactate evolution in the hemodynamic resuscitation progress in early septic shock. Crit Care. 2015;19:126.

21. Shaban M, Salahuddin N, RaedKolko M, Sharshir M, AbuRageila M, Al Hussain A. The predictive ability of $\mathrm{Pv}-\mathrm{aCO}_{2}$ gap and $\mathrm{Pv}-\mathrm{aCO}_{2} / \mathrm{Ca}-\mathrm{vO}_{2}$ ratio in shock: a prospective, cohort study. Shock. 2017;47:395-401.

22. Knaus W, Draper E, Wagner DP. Zimmerman JE. APACHE II: A severity of disease classification system. Crit Care Med. 1985;13:818-29.

23. Vincent JL, de Mendonça A, Cantraine F, Moreno R, Takala J, Suter P, Sprung C, Colardyn F, Serge B. Use of the SOFA score to assess the incidence of organ dysfunction/failure in intensive care units: Results of a multicenter, prospective study. Crit Care Med. 1998;26:1793-800.

24. Faubel S, Patel NU, Lockhart ME, Cadnapaphornchai MA. Renal Relevant Radiology: Use of Ultrasonography in Patients with AKI. Clin J Am Soc Nephrol. 2014;9:382-94.

25. Singer M, Deutschman CS, Seymour CW, Shankar-Hari M, Annane D, Bauer M, et al. The Third International Consensus Definitions for Sepsis and Septic Shock (Sepsis-3). JAMA. 2016;315:80110.

26. Cecconi M, De Backer D, Antonelli M, Beale R, Bakker J, Hofer C, et al. Consensus on circulatory shock and hemodynamic monitoring. Task force of the European Society of Intensive Care Medicine. Intensive Care Med. 2014;40:1795-815.

27. Kellum JA, Lameire N. Diagnosis, evaluation, and management of acute kidney injury: a KDIGO summary (Part 1). Crit Care. 2013;17:204.

28. Oliveira RAG, Mendes PV, Park M, Taniguchi LU. Factors associated with renal Doppler resistive index in critically ill patients: a prospective cohort study. Ann Intensive Care. 2019;9:23.

29. Anile A, Ferrario S, Campanello L, Orban MA, Castiglione G. Renal resistive index: a new reversible tool for the early diagnosis and evaluation of organ perfusion in critically ill patients: a case report. Ultrasound J. 2019;11:23. 
30. Darmon M, Schortgen F, Leon R, Moutereau S, Mayaux J, Di Marco F, Devaquet J, Brun-Buisson C, Brochard L. Impact of mild hypoxemia on renal function and renal resistive index during mechanical ventilation. Intensive Care Med. 2009;35:1031-8.

31. Mallat J, Lemyze M, Meddour M, Pepy F, Gasan G, Barrailler S, et al. Ratios of central venous-toarterial carbon dioxide content or tension to arteriovenous oxygen content are better markers of global anaerobic metabolism than lactate in septic shock patients. Ann Intensive Care. 2006;6:10.

32. Teboul JL, Saugel B, Cecconi M, De Backer D, Hofer CK, Monnet X, et al. Less invasive hemodynamic monitoring in critically ill patients. Intensive Care Med. 2016;42:1350-9.

33. Bellomo R, Ronco C, Mehta RL, Asfar P, Boisrame-Helms J, Darmon M, et al. Acute kidney injury in the ICU: from injury to recovery: reports from the 5th Paris International Conference. Ann. Intensive Care. 2017;7:49.

34. Hoste EA, Bagshaw SM, Bellomo R, Cely CM, Colman R, Cruz D, et al. Epidemiology of acute kidney injury in critically ill patients: the multinational AKI-EPI study. Intensive Care Med. 2015;41:1411-23.

35. Ronco C, Bellomo R, Kellum AJ. Acute kidney injury. Lancet. 2019;394:1949-64.

36. Calabia J, Torguet P, Garcia MD, Martin I, Mate N, Marin G, Molina A, Valles C. M. The relationship between renal resistive index, arterial stiffness, and atherosclerotic burden: the link between macrocirculation and microcirculation. The Journal of Clinical Hypertension. 2014;16:186-91.

\section{Figures}


(a)

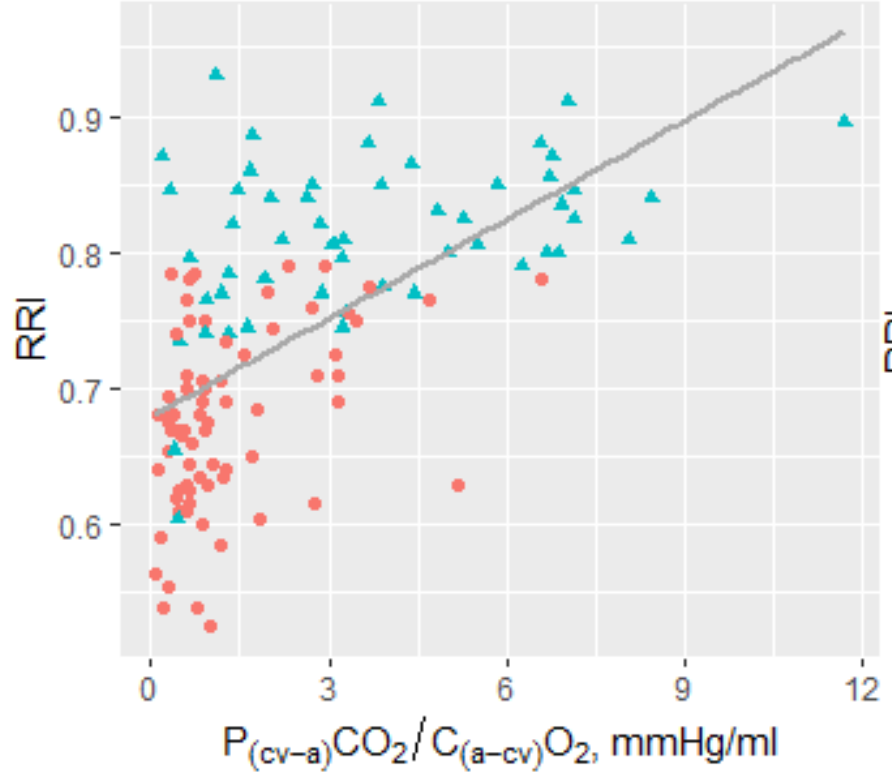

(c)

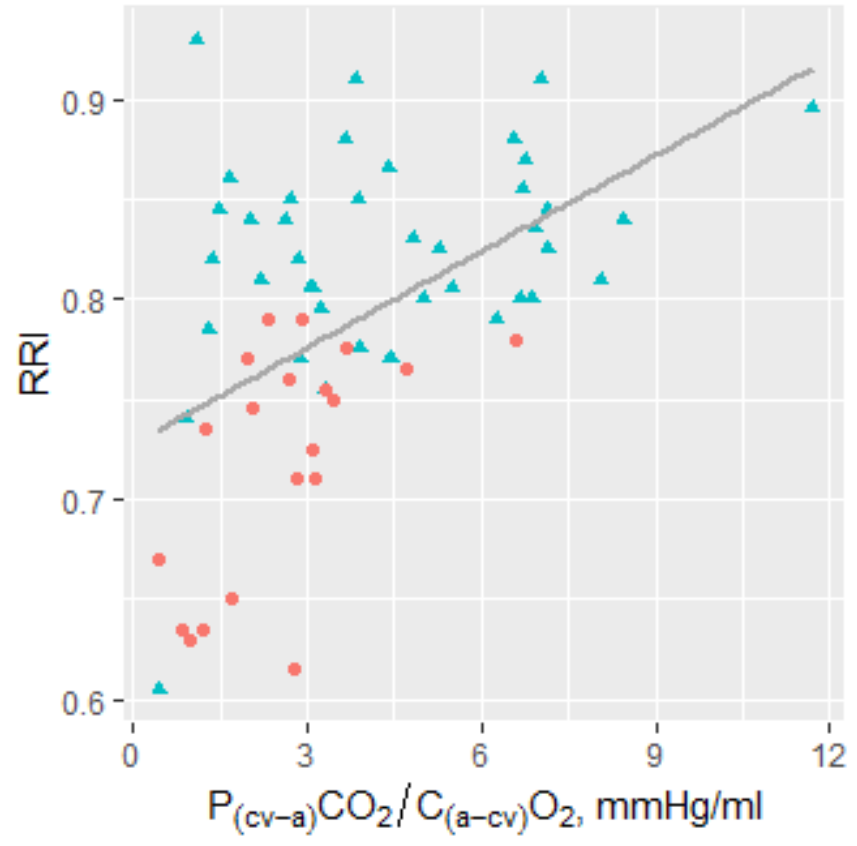

(b)

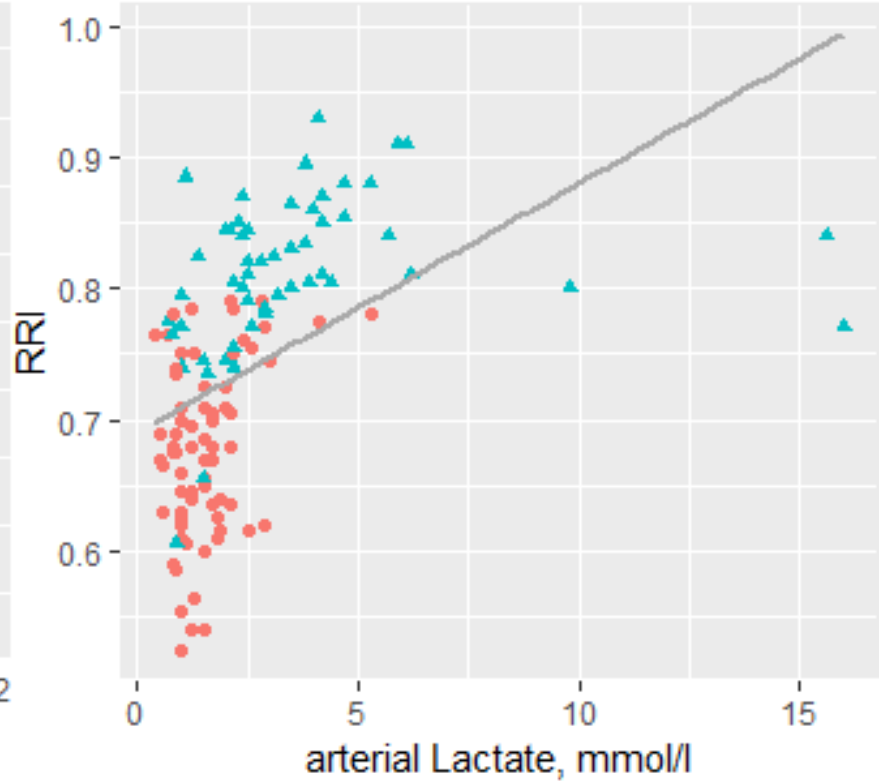

(d)

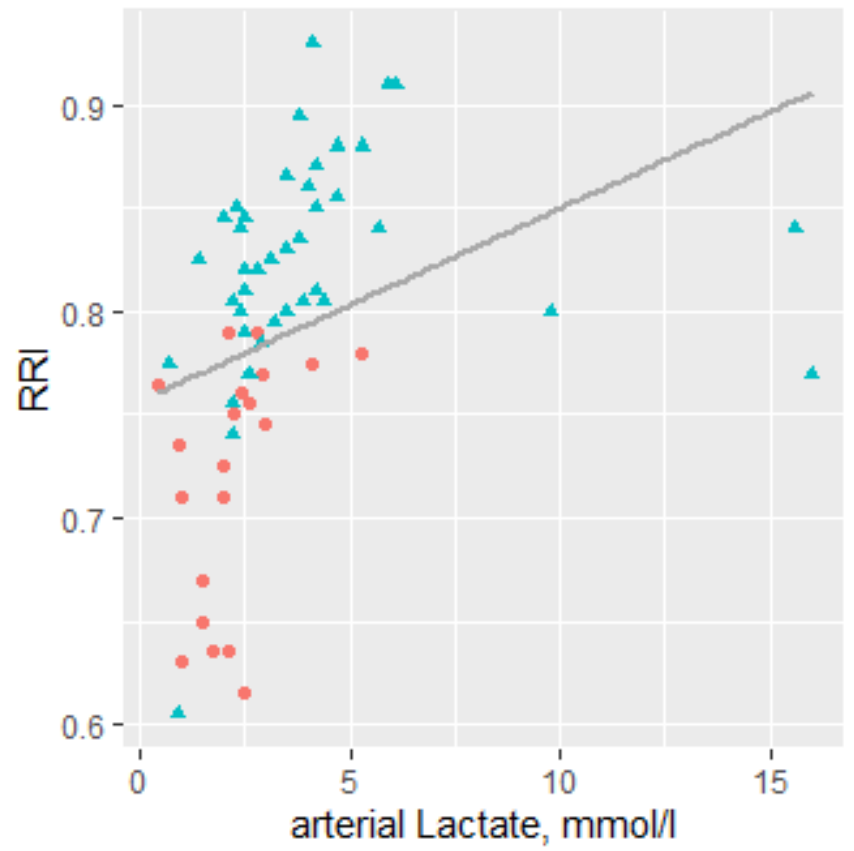

\section{Figure 1}

Correlation scatter plot for $\mathrm{RRI}$ versus $\mathrm{P}(\mathrm{cv}-\mathrm{a}) \mathrm{CO} 2$ / $\mathrm{C}(\mathrm{a}-\mathrm{cv}) \mathrm{O} 2$ ratio and arterial lactate in patients with (triangles) and without (dots) acute kidney injury (AKI) development, for the total patient population (3a and $3 b$, respectively, rho $=0.64, p<0.0001$, in both cases) as well as for the subset of patients with shock ( $3 c$ and $3 d$, rho $=0.48, p=0.001$ and rho $=0.64, p<0.001$, respectively) 


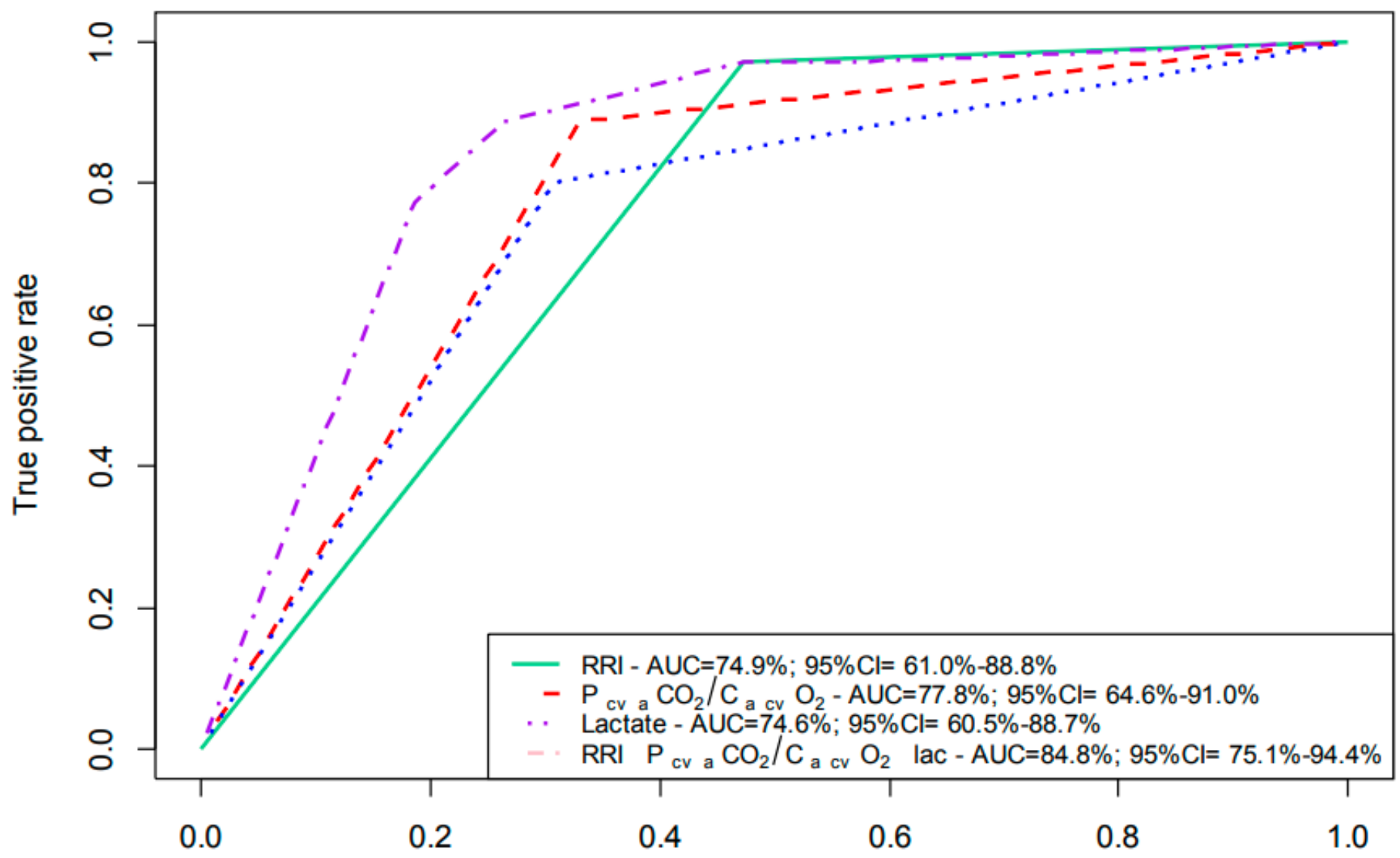

False positive rate

Figure 2

Receiver-operating characteristic curves showing the ability of renal resistive index (RRI), the ratio of central venous-to-arterial carbon dioxide partial pressure difference by arterial-to-central venous oxygen content difference $(\mathrm{P}(\mathrm{cv}-\mathrm{a}) \mathrm{CO} 2$ / $\mathrm{C}(\mathrm{a}-\mathrm{cv}) \mathrm{O} 2)$ ratio, arterial lactate and the combination of them, with area under the curve $(\mathrm{AUC})$ and confidence interval $(\mathrm{Cl})$ values, to predict ICU mortality. 